\title{
Ondas Ultrassônicas: teoria e aplicações industriais em ensaios não-destrutivos
}

Douglas Morais

douglas.morais2@fatec.sp.gov.br Faculdade de Tecnologia de Itaquera (FATEC), São Paulo, São Paulo, Brasil.

Daniel Lyra Rodrigues

daniel lyra@terra.com.br

Universidade de Mogi das Cruzes UMC) Campus Villa Lobos, São Paulo, São Paulo, Brasil.

Diego Possani

diego possani@msn.com

Universidade de Mogi das Cruzes

(UMC) Campus Villa Lobos, São Paulo,

São Paulo, Brasil.

Fabio Conte Correia

fabiocontee@gmail.com Universidade

de Mogi das Cruzes (UMC) Campus

Villa Lobos, São Paulo, São Paulo,

Brasil.

\section{RESUMO}

Com o aumento da complexidade e do volume de operações nas indústrias de petróleo e mineração, há uma demanda cada vez maior para sistemas integrados de monitoramento de integridade estrutural, que incluem ensaios não destrutivos. Dentro deste contexto, apresenta-se nesse trabalho, por meio de uma investigação teórica, uma breve explanação sobre a viabilidade e flexibilidade da técnica de ultrassom, seguida das bases cientificas e tecnologias desta. Para tal, são apresentados os aspectos teóricos ligados à caracterização das ondas sonoras, a evolução histórica relativa aos estudos sobre ondas sonoras, as principais características da técnica de Ultrassom aplicadas a ensaios não destrutivos e a influência dos materiais nos ensaios de ultrassom. Desta forma, esse trabalho possibilita ao iniciante concluir a importância dessa técnica, além de propiciar os principais conceitos e técnicas.
\end{abstract}

PALAVRAS-CHAVE: Ultrassom; Transdutores ultrassônicos; Ondas acústicas. 


\section{INTRODUÇÃO}

A técnica de ensaio não destrutivo por ultrassom avançou muito nos últimos 20 anos, com o aumento do nível de automação das técnicas disponíveis, diminuindo consideravelmente tempo e custo dos ensaios através da utilização de toda gama possível de propagação de ondas sonoras em materiais isotrópicos e anisotrópicos (BROOK, 2012; CHEEKE, 2002; ROSE, 2004).

A complementação dos testes por ultrassom, com os conhecimentos trazidos pelo desenvolvimento de ensaios de emissões acústicas e novos métodos de tratamento matemático da propagação de ondas sonoras em materiais anisotrópicos, permite expandir ainda mais as fronteiras dessas técnicas, permitindo o ensaio de materiais revestidos ou compósitos (NOGUEIRA, 2009; AGGELIS, 2011-a; AGGELIS, 2011-b).

Esses avanços permitem, às técnicas de inspeção por ondas ultrassônicas, eficácia, flexibilidade e amplitude de aplicações mais abrangentes que qualquer outra técnica de ensaio não destrutivo. A abrangência dessas aplicações permite a essa técnica aproximar-se da situação ideal em relação a uma técnica de ensaio não destrutivo. Assim, observa-se a possibilidade de medição com rapidez, precisão e custo compatível da conformidade de materiais, em momentos cada vez mais próximos à conformação desses nos processos industriais, evitando perdas de produção, desperdício de energia e materiais. Observa-se também a possibilidade de monitoramento contínuo ou medição em campo, até em ambientes agressivos, da integridade estrutural de componentes em serviço, permitindo a expansão de sua vida útil e níveis de segurança de operação.

Assim, nesse contexto, objetiva-se nesse trabalho apresentar as bases cientificas e tecnologias da técnica de ultrassom, onde são apresentados aspectos teóricos ligados à caracterização das ondas sonoras, a evolução histórica relativa aos estudos sobre ondas sonoras, as principais características da técnica de Ultrassom aplicadas a ensaios não destrutivos e a influência dos materiais nos ensaios de ultrassom.

\section{CARACTERIZAÇÃO FÍSICA DE ONDAS SONORAS}

Ondas sonoras são ondas mecânicas, ou seja, correspondem fisicamente à propagação de energia através de materiais. Por isso, velocidade de propagação do som é diferente nos diversos tipos de materiais e varia de acordo com as características dos materiais, tais como densidade, rigidez e organização estrutural (CHEEKE, 2002; ROSE, 2004; LUZ e ÁLVARES, 2005; NUSSENZVEIG, 2002).

As características particulares da estrutura de material determinarão também a maneira como as ondas mecânicas se propagarão. Essas ondas podem se propagar por diversos modos, tendo cada um sua velocidade de propagação definida conforme as equações 1.1 a 1.5 listadas abaixo,

Velocidade de Onda Longitudinal:

$$
v L=\sqrt{\frac{E}{\rho} \cdot \frac{1-\mu}{(1+\mu) \cdot(1-2 \mu)}}
$$


Velocidade de Onda Transversal:

$$
v T=\sqrt{\frac{E}{\rho} \cdot \frac{1}{2(1+\mu)}}=\sqrt{\frac{G}{\rho}}
$$

Velocidade de Onda de Dilatação:

$$
v D=\sqrt{\frac{E}{\rho}}
$$

Velocidade de Onda de Flexão:

$$
v B=\frac{2 w}{\lambda} \sqrt{\frac{E}{\rho} \cdot \frac{I}{F}}
$$

Velocidade de Onda Superficial:

$$
v O=\frac{0,87+1,12 \mu}{1-\mu} \cdot v T
$$

em que $\lambda=$ comprimento de onda mecânica, $\mu=$ coeficiente de Poisson, $\rho=$ densidade do material, $\mathrm{E}=$ módulo de Young ou módulo de elasticidade, $\mathrm{G}=$ módulo de rigidez ou de cisalhamento, $w$ = largura da placa ou barra, I = momento de inércia e $\mathrm{F}=$ determinante de dispersão, obtido a partir da matriz de transformada de dispersão (BOLLER, 2009).

Uma primeira classificação desses modos está relacionada aos planos de propagação da onda sonora, e é dependente da rigidez do material (BOLLER, 2009; M ARTIN, 2012).

MARTIN (2012) propôs um modelo simplificado da estrutura de um material utilizando esferas e molas, onde as esferas representam as partículas e as molas, as forças de atração e repulsão entre elas. Considerando os módulos de rigidez elástica $(E)$ e de rigidez ao cisalhamento $(G)$ como a rigidez das molas que unem as partículas do modelo, respectivamente à compressão/tração e rotação é possível entender a relação direta da velocidade de propagação de onda sonora com os módulos de rigidez elástica (E) e de cisalhamento (G) e o coeficiente de Poisson ( $\mu$ ) (BOLLER, 2009; M ARTIN, 2012).

Um ou mais dos tipos de propagação de ondas sonoras, podem ocorrer simultaneamente em um mesmo material. Uma frente de onda longitudinal, que é mais veloz, pode ser seguida por uma frente de onda transversal, que é mais lenta. Um dos objetivos das técnicas de ultrassom é excitar tipos diferentes de ondas em um material, obtendo-se através do modo de propagação de cada uma delas, conclusões a respeito das características da estrutura e defeitos deste material (CHEEKE, 2002; MARTIN, 2012).

Em líquidos e gases, os modos de propagação de onda sonora, que incluem 0 movimento perpendicular ao sentido de propagação da onda, como em ondas transversais, de flexão e superficiais são atenuados e praticamente inexistem nesses estados, devido à baixíssima rigidez ao cisalhamento nesses estados (CHEEKE, 2002; ROSE, 2004).

Nos materiais sólidos, ou em líquidos com alta viscosidade, a identificação dos diferentes tipos de propagação de ondas sonoras e de seu nível de intensidade pode trazer evidências de defeitos, níveis de dureza e até sentidos de organização e crescimento granular (BROOK, 2012; M ARTIN, 2012). 


\section{EVOLUÇÃO HISTÓRICA DOS ESTUDOS SOBRE ONDAS SONORAS}

Na segunda metade do século XVII, com o desenvolvimento do pensamento cartesiano e a estruturação das cadeiras universitárias, tanto de ciências naturais e quanto de tecnologia mecânica, foi possível estruturar as primeiras teorias em relação à propagação das ondas sonoras no ar e em materiais utilizados pela engenharia. Neste momento, pela primeira vez a ciência catalogava as evidências de existência de frequências sonoras fora da faixa audível pelo ouvido humano (entre $20 \mathrm{~Hz}$ e $20 \mathrm{kHz}$ ) e começava a sistematizar métodos que utilizavam o som como instrumento de detecção, medição ou alerta (CHEEKE, 2002).

Nessa época, o estudo da audição mais apurada de animais como o morcego, criou os primeiros indícios de ultrassom, ou seja, propagação de ondas sonoras com frequência acima do limiar do ouvido humano $(20 \mathrm{kHz})$. Os estudos relacionados à sismologia, mecânica da fratura e vibrações mecânicas catalogavam as primeiras evidências do infrassom, ou seja, da propagação de ondas sonoras com frequência abaixo do limiar do ouvido humano $(20 \mathrm{~Hz})$ (CHEEKE, 2002). Com a revolução industrial, técnicas milenares foram finalmente entendidas, descritas e sistematizadas, como o ensaio de tinido, utilizado desde antiguidade por ferreiros, na determinação da dureza de um material em processo metalúrgico, pelo som emitido pelas batidas do martelo que conforma o material metálico (M ARTIN, 2012).

Ainda que demorasse até o final do século XIX, para que o desenvolvimento da teoria da piezeletricidade permitisse a construção de sensores e emissores de infra e ultrassom, a base matemática para descrição da propagação de ondas sonoras já estava consolidada por Rayleigh e Fourier, na metade do século XVIII (M ARTIN, 2012; TELLES, 2010).

O naufrágio do Titanic e a corrida armamentista para a Primeira Guerra M undial criaram justificativas comerciais para o desenvolvimento dos primeiros sonares, equipamentos que permitiam a detecção à longa distância de submarinos inimigos, icebergs e outros obstáculos naturais, abaixo da superfície da água, através da emissão de ondas ultrassônicas e da interpretação do seu eco (M ARTIN, 2012).

A tecnologia dos sonares, pelo fato dos sonares dedicarem-se à emissão e detecção de ondas sonoras na água ou ar, utilizou apenas a propagação de ondas sonoras longitudinais.

Na década de 1950, com a consolidação e difusão dos conhecimentos sobre mecânica da fratura, os quais deram um grande salto tecnológico nas duas décadas anteriores, foram construídos, na então União Soviética e Alemanha, os primeiros equipamentos de detecção de defeitos em materiais através da emissão de ondas ultrassônicas, focando principalmente defeitos em materiais metálicos e suas uniões soldadas (M ARTIN, 2012; TELLES, 2010).

A partir desta utilização, ganha importância e uma descrição muito mais apurada, o estudo da propagação de ondas sonoras transversais. Cabeçotes de emissão de ondas longitudinais são então adaptados para a emissão e recepção deste tipo de onda sonora, o que corresponde basicamente à articulação geométrica da posição do cabeçote ultrassônico em relação à superfície do material ensaiado (MARTIN, 2012). 
As leis de reflexão e refração de ondas sonoras, derivadas dos estudos de propagação de ondas luminosas da ótica, tornam-se a base matemática para a separação do volume de ondas transversais e longitudinais, propagando simultaneamente em um material sólido ou líquido com alta viscosidade. A mudança de uma onda sonora de longitudinal para transversal, ou vice-versa, na sua propagação ao longo de um material chama-se modo de conversão (CHEEKE, 2002).

0 modo de conversão de ondas sonoras é determinado principalmente pelo ângulo de incidência, também chamado de ataque. Com a variação desse ângulo é possível controlar-se o volume de ondas longitudinais e transversais produzidas (BROOK, 2012).

Em uma faixa estreita em torno de 65 graus de ângulo de incidência, há a geração de um terceiro tipo de ondas ultrassônicas, chamada de superfície. 0 uso das ondas de superfície originará outra técnica de ultrassom, chamada de ondas guiadas, mas tal tecnologia somente começou a ter um uso comercial mais difundido a partir do começo da década de 1980 (CHEEKE, 2002; ROSE, 2004; OLYM PUS, 2007).

A geometria dos elementos inspecionados e mudanças em sua composição interna, também influenciam modos de conversão secundários. 0 domínio do conhecimento desses modos de conversão é crítico para muitas aplicações, como a inspeção de soldas, uma vez que determina o posicionamento dos transdutores para recepção dos ecos ultrassônicos (M ARTIN, 2012).

Até esse ponto na história, todas as técnicas de ensaio por ultrassom estão no nível atualmente conhecido como técnica de pulso-eco e visualização por AScan, ou seja, emissão de ondas longitudinais ou transversais através de um cristal piezelétrico, em uma superfície e detecção dessas ondas em uma superfície oposta à superfície de emissão, ou até na mesma superfície de emissão, em um ponto igual ou diferente da emissão (BROOK, 2012; CHEEKE, 2002; M ARTIN, 2012).

A visualização por A-Scan na técnica de ultrassom por pulso-eco é a interpretação da geometria interna de um material através do tempo de chegada das frentes de ondas longitudinais e transversais ao cabeçote ultrassônico de detecção (representadas por picos na tela). Como as ondas transversais propagam-se em velocidades menores às ondas longitudinais, conhecendo-se os modos de conversão relativos à geometria da peça e posicionamento do transdutor, é possível inferir sobre os tipos de defeitos, sua geometria, tamanho e localização (BROOK, 2012; CHEEKE, 2002; M ARTIN, 2012).

Transdutores ultrassônicos começaram a ser combinados em diferentes ângulos de incidência, para a determinação mais precisa de defeitos, sua geometria e tamanho, pois, com a combinação dos ângulos de incidência, tornase possível a varredura de uma área maior a ser examinada, possibilitando uma melhor determinação do local e tamanho da trinca que se está procurando (BROOK, 2012).

Os ângulos de ataque e fuga de diferentes cabeçotes ultrassônicos pela técnica pulso-eco, não somente determinam os modos de conversão de ondas longitudinais para transversais, como também podem ser articulados tridimensionalmente, para gerar imagens ultrassônicas bidimensionais, chamadas 
de B-Scan e D-Scan, e tridimensionais, chamadas de C-Scan e S-Scan (CHEEKE, 2002; M ARTIN, 2012).

Torna-se cada vez mais comum a comercialização de aparelhos de ultrassom com mais de um modo de visualização. Com as visualizações B-Scan e C-Scan, torna-se possível a consolidação comercial da segunda técnica de ultrassom, conhecida como ToFD (Time of Flight Diffraction), ou seja, a interpretação do tempo de percurso não somente de ondas refletidas como na técnica pulso-eco, mas também de ondas resultantes da difração por partes geométricas de defeitos, como frentes de propagação de trincas em componentes.

É importante notar que esta técnica é um refinamento da técnica de pulsoeco, ou seja, cabeçotes ultrassônicos de ondas longitudinais e/ou transversais têm seus feixes focados nos limites de defeitos, para determinação mais precisa de seu tamanho e orientação (M ARTIN, 2012).

A partir da década de 1960, as aplicações comerciais do ultrassom na medicina, principalmente através do exame de ultrassonografia pré-natal ou do sistema gástrico, criam soluções mais apuradas e diminuem sensivelmente os custos de desenvolvimento e produção dos chamados B-Scan e C-Scan (CHEEKE, 2002).

0 desenvolvimento da computação gráfica na década de 1970 permite uma maior clareza e resolução dos B-Scans e C-Scans, que até então eram apenas composições computacionais de vários A-Scans, articulados tridimensionalmente no ambiente do computador (CHEEKE, 2002).

A queda vertiginosa, do custo, dos componentes eletrônicos piezelétricos, e sua correspondente miniaturização, permite o surgimento nessa década, das primeiras pesquisas da terceira técnica disponível de ultrassom conhecida como Phased Array. Essa técnica consiste na automação de cabeçotes ultrassônicos múltiplos, que em conjunto e mudando sob a programação de um computador, os ângulos de ataque e fuga de ondas ultrassônicas, são capazes de montar BScans e C-Scans de alta resolução, de áreas maiores, em segundos e com o mínimo de movimentação dos cabeçotes de emissão (pitch) e detecção (catch) de ondas ultrassônicas (OLYM PUS, 2007).

0 nível de automação da técnica Phased Array também permite modificar, durante 0 ensaio, a frequência dos cabeçotes múltiplos, sob a programação novamente do computador, permitindo a focalização ultrassônica diferenciada de regiões, ou seja, além de multiplicar a resolução, as possibilidades de detecção e acelerar o tempo dos ensaios, até então feitos pela técnica de pulso-eco, a técnica de Phased Array também traz os mesmos benefícios às aplicações desenvolvidas até então para o ToFD (OLYM PUS,2007; OLYM PUS, 2010; RICHTER, 2013).

A organização dos múltiplos cristais dos transdutores de Phased Array, na forma matricial ou circular aumenta consideravelmente a amplitude de S-Cans e C-Scans, a partir de um mesmo posicionamento do transdutor, acelerando o tempo de inspeção e permitindo o registro de imagens tridimensionais em tempo real, na tela dos aparelhos de ultrassom e computadores conectados (OLYM PUS, 2007).

Apesar de todo desenvolvimento comercial da técnica de Phased Array, na primeira década do século XXI, os custos de aquisição, treinamento e calibração 
ainda são muito altos, se comparados às técnicas pulso-eco e ToFD, mas as necessidades em relação ao desenvolvimento de ensaios de ultrassom em materiais compósitos e para o monitoramento automatizado de materiais em ambientes agressivos tende a aumentar rapidamente a escala de seu uso, diminuindo consequentemente seus custos (OLYM PUS,2007; OLYM PUS, 2010; RICHTER, 2013).

Também é da década de 1970, novamente com o impulso e salto tecnológico das ciências naturais com os estudos sobre sismologia e ecologia que surgiram estudos mais específicos sobre a propagação de ondas superficiais, comuns em abalos sísmicos (BOLLER, 2009). Nesse período iniciou-se também todo o tratamento matemático para aquisição e filtragem de sinais caóticos, simultaneamente nos domínios do tempo e da frequência (ROSE, 2004).

Também neste momento emergiu comercialmente a segunda técnica de ensaio não destrutivo, por ondas acústicas, relegada apenas à sismologia e a laboratórios de estudo de mecânica da fratura desde o início do século XX, trabalhando na faixa do infrassom, chamada emissões acústicas (BOLLER, 2009).

É importante notar que as diferenças entre os ensaios não destrutivos por emissões acústicas e por ultrassom é apenas a ordem de grandeza, tanto das frequências de excitação e captura de ondas sonoras respectivamente de alguns Hertz para alguns milhões de Hertz, quanto na propagação de trincas, que variam respectivamente de alguns milhares de quilômetros em abalos sísmicos a milésimos de milímetro no caso de estruturas e componentes (BOLLER, 2009).

Os ensaios acústicos estão presentes desde os primórdios da humanidade, mesmo que de forma intuitiva. Os primeiros ensaios baseavam-se na simples atenção ao ouvir o ranger de uma viga ou pilar de madeira, seja em um mastro ou viga de barco, viga de uma casa, ponte, ou árvore, o que significava a propagação de uma ou mais trincas, já próximo ao limiar da resistência da madeira (CHEEKE, 2002). Baseavam-se também na atenção ao brilho acústico, isto é, ao som emitido pelos metais tratados termicamente durante sua conformação ou na identificação de materiais de diferentes composições (CHEEKE, 2002).

Com o passar do tempo e o aumento da necessidade da previsão de falhas, os ensaios acima citados tiveram por necessidade evoluir. Com isso, o primeiro ensaio deu origem ao ensaio de emissões acústicas, que permite a detecção de microtrincas devido à captação do infrassom. 0 segundo foi substituído pelo ensaio de ultrassom, devido a sua incapacidade de detectar trincas no meio dos materiais.

No começo da década de 1980, o controle sobre a interpretação e emissão de ondas superficiais cria, comercialmente, a quarta técnica de ensaio de ultrassom disponível atualmente, chamada de Ondas Guiadas, que corresponde à emissão de ondas paralelamente às superfícies ensaiadas. Pelo baixo índice de atenuação deste tipo de onda, é possível inspecionar áreas de material, com até algumas centenas de metros de distância entre os transdutores ultrassônicos emissores e receptores (ROSE, 2004).

Essa técnica somente tornou-se possível também através do conhecimento e tecnologia acumulados pelas técnicas anteriores: 
- pulso-eco: da qual se extraiu o conhecimento relacionado à atenuação de ondas de diferentes frequências em cada material e ângulos limites de ataque e fuga, para geração de ondas superficiais através de transdutores ultrassônicos de ondas transversais;

- ToFD: da qual se obteve o conhecimento em relação à difração de ondas sob obstáculos com diferentes tamanhos e disposições geométricas;

- Phased Array: que deu origem a tecnologia e ao conhecimento para a emissão e controle de ondas que são chamadas de guiadas, pois realmente tem seu percurso ao longo de uma superfície coordenado pela concatenação de transdutores ultrassônicos múltiplos, automatizados por computador.

Existem dois tipos de ondas superficiais. As ondas de Rayleigh que correspondem a ondas superficiais em materiais ou meios de espessura com ordem de grandeza superior em muitas vezes, em relação ao seu comprimento, 0 que corresponderia ao que chama de onda superficial (BOLLER, 2009; MARTIN, 2012). E as ondas de Lamb Simétricas e Assimétricas que correspondem a ondas superficiais em materiais ou meios de espessura com ordem de grandeza inferior, em muitas vezes, em relação ao comprimento da onda. As ondas de Lamb podem ainda subdividir-se em ondas simétricas e assimétricas, o que corresponderia respectivamente às denominações de ondas de flexão e dilatação, propostas por (BOLLER, 2009).

0 último tipo de onda utilizado pelas técnicas de ultrassom é conhecido por ondas de Creeping, que é um tipo de onda superficial que difere um pouco da onda de Rayleigh. As técnicas de ensaio que utilizam ondas de Creeping, têm como objetivo a identificação de corrosão na superfície interna ou externa de uma peça, fornecendo estimativa da extensão da área e da espessura nominal (M ARTIN, 2012) e a inspeção de soldas de aço austenístico (MARTIN, 2012).

Todo o trabalho de desenvolvimento tecnológico e comercial sobre ondas superficiais traz dois avanços adicionais importantes, resultantes do fechamento de um ciclo de conhecimento, que engloba todos os tipos de propagação de ondas mecânicas:

- maior conhecimento e técnicas conjugadas de medição por ultrassom de materiais compósitos, principalmente com organização macroscópica em várias camadas (ROSE, 2004; AGGELIS, 2011-b);

- maior conhecimento e técnicas conjugadas de medição por ultrassom sobre a mecânica da fratura não somente para metais e componentes de pequeno porte, mas para estruturas de centenas de metros de dimensão e construídas também por materiais compósitos, até de matriz cimentícia e que não são organizados macroscopicamente em camadas (NOGUEIRA, 2009; AGGELIS, 2011a).

No final da década de 1990, consolida-se comercialmente principalmente na técnica de ondas guiadas, a utilização de um novo tipo de tecnologia de transdutor ultrassônico, que não funciona através da excitação de materiais piezelétricos, mas através da excitação de ondas ultrassônicas, por força eletromagnética. Esses transdutores recebem o nome de Eletro Magnetic Acoustic Transducer (EM AT), ou transdutores acústicos eletromagnéticos (HIRAO $\&$ OGI, 2003). 
0 princípio de funcionamento dos transdutores acústicos eletromagnéticos se dá pela aproximação do transdutor ao material que se quer ser examinado. 0 transdutor possui um ímã e um circuito com uma bobina, que ao ser aproximado ao material, gera um campo magnético, que por sua vez gera ondas ultrassônicas (HIRAO \& OGI, 2003).

Os transdutores eletromagnéticos possuem capacidade para gerar ondas longitudinais, transversais e superficiais. A grande vantagem dos transdutores acústicos eletromagnéticos em relação aos de materiais piezelétricos é a ausência de contato da superfície do transdutor com a superfície inspecionada, o que permite a inspeção de superfícies com temperaturas superiores a $125^{\circ} \mathrm{C}$ (HIRAO $\&$ OGI, 2003).

Este é o cenário atual, da metade da segunda década do século XXI, onde técnicas de emissões acústicas unem-se tanto na teoria como na prática a técnicas de ultrassom, na manutenção da integridade e estabilidade de componentes e estruturas dos mais diversos materiais (BOLLER, 2009).

\section{EVOLUÇÃO DOS TRANSDUTORES ULTRASSÔNICOS E SEUS CABEÇOTES DE INSPEÇÃOO}

Para cada uma das quatro técnicas de ultrassom disponíveis: pulso-eco, ToFD, phased-array e ondas guiadas, 0 posicionamento dos transdutores ultrassônicos e a forma de contato com a superfície do componente inspecionado são as características mais críticas para a determinação de quais tipos de ondas sonoras atravessam o material inspecionado, bem como a amplitude e profundidade do volume examinado pelo ensaio (BROOK, 2012).

0 posicionamento dos transdutores ultrassônicos e a forma de contato com a superfície do componente inspecionado evoluiu para um conjunto de componentes denominado cabeçote ultrassônico.

As normas sobre ultrassom denominam cabeçote ultrassônico à composição de elementos formada pelo conjunto de transdutores, fluidos ou lentes acoplantes do transdutor à superfície examinada e dispositivos de fixação e translação dos transdutores.

\section{TRANSDUTORES ULTRASSÔNICOS}

Os transdutores ultrassônicos utilizam a deformação dos cristais piezelétricos tanto para gerar energia sônica no elemento examinado, quanto para coletar 0 restante de energia sônica que atravessou o elemento examinado e não foi dissipado por esse.

Em cada uma das quatro técnicas disponíveis de ultrassom, dependendo do nível de precisão da medição e da possibilidade de acesso ao material inspecionado, podem ser selecionados:

- transdutores exclusivos de emissão, também chamados de pitch, ou seja, energia sônica a este; 
- transdutores exclusivos de recepção, também chamados de catch, ou seja, esses transdutores somente serão excitados por ondas sonoras que atravessaram o material examinado ou que foram refletidas por esse, no caso do transdutor receptor encontrar-se na mesma superfície que o transdutor emissor;

- transdutores de cristal piezelétrico duplo, os quais possuem dois cristais piezelétricos montados em uma mesma carcaça, configurando o mesmo ponto de emissão e recepção (MARTIN, 2012).

Todos os transdutores podem ser excitados em uma frequência determinada pelo equipamento de ultrassom ou simplesmente serem deixados em contato com a peça examinada, à espera de ondas sonoras refratadas ou refletidas pelo material ao qual está em contato. Dessa forma ainda é possível uma montagem adicional às citadas anteriormente, onde um transdutor de cristal piezelétrico único é excitado pelo aparelho de ultrassom por um determinado momento e deixado em repouso em um intervalo programado seguinte. Dessa forma o transdutor é capaz de realizar ciclos combinados de emissão de ondas sonoras e leitura de seu eco na mesma posição (BROOK, 2012).

Os transdutores de Phased Array podem ser coordenados por um programa de computador para varreduras distintas, substituindo o trabalho de cabeçotes simples e duplos de monocristal, criando a possibilidade de detecção mais eficaz de tipos diferentes de defeitos. Os tipos de varredura mais comuns de Phased Array, pela interpolação da ativação dos múltiplos cristais piezelétricos encontram-se na Figura 1, abaixo (OLYM PUS, 2007).

Figura 1- Modos de varredura através da interpolação da ativação dos transdutores múltiplos de um cabeçote (Phased Array)

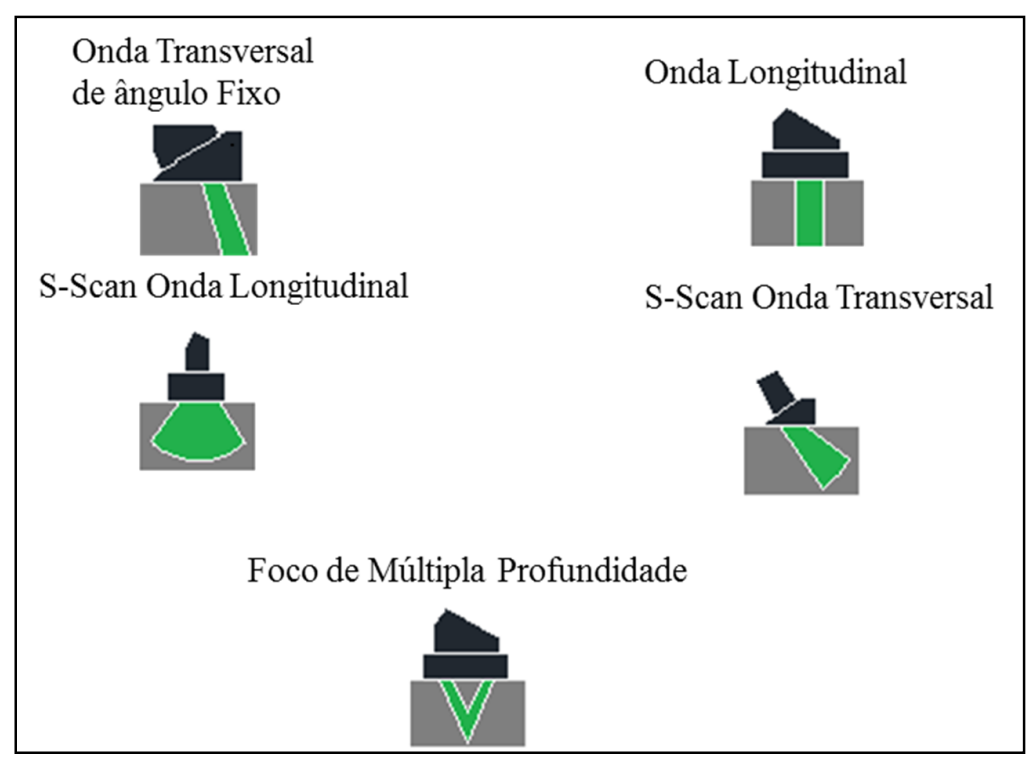

Fonte: adaptada pelos autores a partir de Olympus (2007, p.23).

Os transdutores de Phased Array são capazes, portanto, de serem coordenados por programas computacionais pré-definidos de varredura, em um componente a ter suas características verificadas por um ensaio não-destrutivo, permitindo a automação dos procedimentos de ensaio. 


\section{BLOCOS DE REFERÊNCIA, LENTES E FLUIDOS ACOPLANTES}

As principais funções do fluido ou lentes acoplantes são, de acordo com Olympus (2007), :

- transferir a energia sônica produzida pelo cristal piezelétrico do transdutor ultrassônico com o mínimo de atenuação ou distorção;

- assegurar os ângulos de incidência aos modos de conversão desejados para ondas longitudinais, transversais e superficiais;

- proteger a superfície do transdutor do desgaste relativo ao atrito com a superfície examinada;

- adaptar a superfície do transdutor que possui curvatura, rugosidade e faixa de temperaturas de serviço específicas, às mesmas condições, nos componentes examinados.

Os fluidos e lentes acoplantes são projetados para mínima perda de energia sônica entre o transdutor e o componente examinado. Os fluidos e lentes mais simples, disponíveis comercialmente, apresentam perda de intensidade acústica de no máximo $2 \%$. Fluidos e lentes acoplantes também são desenvolvidos para acomodar os transdutores a superfícies de componentes que não são totalmente planas e com diferentes tipos de acabamento superficial.

Outro tipo de acessório essencial ao trabalho dos transdutores ultrassônicos são os blocos de referência, que podem ser normalizados ou desenvolvidos sob medida para aplicações especiais (BROOK, 2012).

Blocos de referência são construídos no mesmo material dos componentes inspecionados e possuem características geométricas e defeitos simulados, que tem o objetivo de servir de referência ao aparelho de ultrassom e operador, no início de cada inspeção. São elementos essenciais principalmente para os equipamentos de ultrassom mais simples com apenas modos de visualização AScan ou B-Scan. Servem também para a avaliação de integridade do cristal piezelétrico no início de cada inspeção (BROOK, 2012).

Para superfícies curvas, comuns em tubulações e vasos de pressão, podem ser desenvolvidas lentes especiais, que se ajustam ao raio de curvatura, da superfície inspecionada, sem distorcer os ângulos de incidência. A inovação mais recente neste campo são os cabeçotes de Phased Array flexíveis, que se ajustam a uma gama maior de raios de curvatura, permitindo a inspeção em diferentes partes de um vaso de pressão, sem troca da lente ou transdutor (RICHTER, 2013).

\section{DISPOSITIVOS DE FIXAÇÃO E TRANSLAÇÃO}

Os dispositivos de fixação e translação de transdutores possuem a função de manter a consistência do posicionamento dos transdutores em relação à superfície de inspeção. Por consistência desse posicionamento entende-se que (BROOK, 2012):

- a manutenção da geometria de posicionamento dos transdutores emissores e coletores do feixe ultrassônico, ou seja, o ponto focal e amplitude do feixe ultrassônico emitido tem de coincidir com o ponto focal e amplitude do feixe ultrassônico coletado, sob pena de perda, completa ou parcial, da intensidade 
das ondas emitidas pelo transdutor ou refletidas e difratadas pelos defeitos do material;

- a referência da posição inicial de inspeção, no componente ou estrutura examinado, já que na grande maioria das aplicações, na imagem ultrassônica, seja por A-Scan, B-Scan ou até C-Scan, não é possível identificar pontos de referência, reconhecidos visualmente, já que por questões econômicas ou de acesso, não há como inspecionar todas as superfícies de um elemento. Tal erro é comum até em inspeções de grande porte, em vasos de pressão ou estruturas offshore;

- a manutenção da distância e ângulo entre o transdutor e o plano da superfície inspecionada, fator que determina, a partir de leis derivadas da ótica (Lei de Snell), os ângulos de ataque e fuga do feixe ultrassônico e consequentemente os tipos de ondas sonoras geradas, suas parcelas no volume total do feixe ultrassônico e seu foco;

- a manutenção de características homogêneas de acoplamento entre o transdutor e as superfícies do material examinado durante o teste, ou seja, mesmo que se utilizem os transdutores mais modernos, de contato direto, que não necessitam de fluido acoplante ou que são flexíveis, e que se ajustam à curvatura da superfície examinada, há de se considerar que toda superfície possui um nível de rugosidade que pode variar, e cada aplicação de inspeção possui uma faixa de temperaturas de serviço. Mudanças nas condições de rugosidade e temperatura podem afetar a velocidade e tipo das ondas sonoras, interferindo nas conclusões dos exames realizados.

A translação dos transdutores pode ser automatizada ao sistema de aquisição de sinais ultrassônicos, proporcionando a emissão de relatórios de inspeção em menos tempo e menos vulneráveis a erros de referência, relacionados ao ponto zero ou início da inspeção. Essa automatização, unida à capacidade dos transdutores de Phased Array em realizar varreduras múltiplas, a partir de um único ponto de inspeção, diminui sensivelmente os tempos de inspeção nos processos de fabricação e em campo (ARIAUDO, 2013).

\section{INFLUÊNCIA DOS MATERIAIS NOS ENSAIOS DE ULTRASSOM}

A propagação de ondas ultrassônicas está sempre relacionada aos módulos de rigidez $(G)$ e elasticidade (E) dos materiais em que tais ondas se propagam, uma vez que eles determinam a capacidade de as partículas resistirem à deformação elástica, causada pelo pulso ultrassônico, respectivamente nas direções transversal e longitudinal. Assim como o coeficiente de Poisson, que determina a deformação transversal, resultante da aplicação de uma carga na direção longitudinal (MARTIN, 2012; CHEEKE, 2002). Tais relações ficaram explícitas nas equações 1.1 a 1.5.

Uma vez que as características físicas como densidade, módulo de elasticidade, etc, de cada material são compiladas, torna-se mais fácil notar as diferenças em relação às velocidades de propagação de ondas entre polímeros, metais e materiais compósitos. A faixa de velocidade dos polímeros e poliuretano) está distante da faixa de velocidade dos aços (ASHBY, 2012). 
Tais diferenças podem aumentar ou diminuir dependendo da temperatura de trabalho do material. A cada $50^{\circ} \mathrm{C}$, de diferença, os metais costumam perder cerca de $1 \%$ de velocidade de propagação sônica, já nos polímeros essa perda sobe para 4\% (MARTIN, 2012).

Da mesma forma que há de se considerar tensões adicionais nas junções de dois materiais diferentes, devido a diferentes temperaturas nos processos de fabricação e aplicações em campo, a calibração de equipamentos de ultrassom deve considerar as diferentes condições de temperatura de operação.

Em geral, as velocidades de ondas transversais correspondem de 60 - 70\% da velocidade das ondas longitudinais e a velocidade das ondas superficiais em torno de $90 \%$ da velocidade das ondas transversais. Desse modo, é possível realizar uma interpolação linear dos ábacos anteriores, para a maioria dos materiais mostrados, no caso de ondas transversais e superficiais (M ARTIN, 2012; ASHBY, 2012).

A grande diferença de faixas de velocidade de ondas mecânicas, entre polímeros e metais, e diferentes suscetibilidades dessas velocidades com a mudança de temperatura, traz duas barreiras à aplicação de ultrassom em componentes constituídos por camadas desses materiais.

A primeira barreira está relacionada aos coeficientes de transmissão de ondas mecânicas na interface desses materiais. Quanto maior a diferença entre as velocidades longitudinais de dois materiais, menor será a quantidade de intensidade sonora que será transmitida pela interface.

A frequência na qual o cristal é excitado, seu diâmetro e o material no qual são propagadas as ondas ultrassônicas influenciam diretamente 0 ângulo de abertura no feixe e os comprimentos (N) das zonas críticas do feixe sônico.

Ao atravessar interfaces de materiais com grande diferença de velocidade acústica não somente o feixe sônico perde energia, como também é distorcido em seu ângulo de abertura. Um sistema de ultrassom que lide com camadas sobrepostas de metais e polímeros tem de considerar faixas de frequência onde essas distorções possam ser conhecidas e controladas.

A análise dos ecos ultrassônicos em materiais compósitos ou em componentes formados de múltiplas camadas de materiais, com propriedades físicas muito distintas entre si, foi a última barreira tecnológica a ser ultrapassada, no início dos anos 2000, com a utilização da transformada de wavelets, desenvolvida na década de 1990, substituindo a transformada rápida de Fourier. A vantagem que a transformada de wavelets possui sobre a transformada rápida de Fourier está na flexibilidade de determinação das janelas de tempo para cada faixa de frequência desejada, permitindo a segregação de ecos oriundos de cada camada de material analisado.

Frequências mais altas possuem melhor resolução de tempo e frequências mais baixas possuem melhor resolução de frequência. Resoluções variáveis são sempre benéficas, uma vez que permitem atribuir uma identidade a cada sinal, através dos modos harmônicos, resultando na distinção dos ecos ultrassônicos, por camada do componente e por tipo de material. (BOLLER, 2009 e WEEKS, 2012). 


\section{CONCLUSÃO}

A técnica de inspeção por ultrassom tem sido constantemente aperfeiçoada, tornando-se assim, cada vez mais presente na inspeção de produtos, desde seu processo de conformação, até as fases de manutenção e inspeções, para verificação de sua integridade estrutural, durante todo ciclo de vida de um componente.

A análise do espectro sônico, por técnicas matemáticas desenvolvidas no final da década de 1990, como as transformadas de wavelets, flexibilizaram o processamento de sinais digitais, que anteriormente utilizavam apenas a transformada rápida de Fourier, permitindo a segregação de ecos ultrassônicos, oriundos de camadas distintas de um componente, elaborado em material compósito ou composto de múltiplas camadas de materiais com propriedades físicas muito distintas entre si.

Devido a esses avanços, torna-se cada vez mais viável o estudo do comportamento das ondas sonoras na transição entre diferentes meios, tornando possível a inspeção de tubulações metálicas revestidas por polímeros, utilizadas nas indústrias de petróleo e mineração. 


\title{
Ultrasonic Waves: Theory and Industrial Applications in Non-destructive Testing
}

\begin{abstract}
With the complexity and operations' volume of mining and oil industries increasing, there will be a greater demand for Integrated monitoring of structural integrity, that include non-destructive tests. In this context, this work aims to present the ultrasound that is one of the most important technique to Improve this monitoring. The history, scientific and technology bases of this technique as well the theoretical and practical aspects for characterizing sound waves will be correlated with their use for non-destructive test, associated with the characteristic properties of each material. Therefore, this work allows the beginner to conclude the importance and main concepts that the ultrasound technique.
\end{abstract}

KEYWORDS: Ultrasound; Ultrasonic transducers; Ultrasonic waves. 


\section{Las Ondas Ultrasónicas: Teoria y Aplicaciones Industriales en Ensayos No Destructivos}

\section{RESUMEN}

Con el aumento de la complejidad y del volumen de las operaciones en las industrias de petróleo y mineración, crece la demanda por proyectos avanzados de monitoramiento de la intregridad estructural, los cuales incluyen ensayos no destructivos. En ese contexto este trabajo pretende presentar las bases científicas y tecnologícas de las técnicas de ultrasonido, en la que son presentados los aspectos teóricos relacionados a la caracterización de las ondas sonoras; la evolución histórica de los estudios de las ondas sonoras; las principales características de las técnicas de ultrasonido aplicadas a los ensayos no destructivos y la influencia de los materiales en los ensayos de ultrasonido. De esta forma proporcionando al iniciante en el área los principales conceptos teóricos.

PALABRAS CLAVE: Ultrasonido; Transductores ultrasónicos; Ondas acústicas. 


\section{REFERÊNCIAS}

ABENDI, Associação Brasileira de Ensaios Não Destrutivos e Inspeção - Guia de END e Inspeção \& Programa de Atividades 2013. Editora ABENDI, 1a edição, São Paulo , 2013.

AGGELIS, D.G. Advanced Ultrasonic Characterization of Fresh Cementitous Materials. Editora Nova Science Publishers, 1a edição, 73 p., Nova York, 2011-a.

AGGELIS, D. G. Characterization of Advanced Structural Materials by Acoustic Emission Indices. Editora Nova Science Publishers, 1a edição, 53 p., Nova York, 2011-b.

ARIAUDO, R. Centro de Tecnologia e Construção Offshore Saipem do Brasil no Guarujá. In: Apresentação ao Comitê do Setor de Óleo e Gás da Associação Brasileira de Ensaios Não-Destrutivos e Inspeções, 2013.

ASHBY, M. Seleção de Materiais no Projeto Mecânico. Editora Campus, ed. 4 (tradução), São Paulo, 2012.

BOLLER, C. et al. Enciclopedia of Structural Health Monitoring. Ed. John Wiley and Sons, 1a edição, Nova York, 2009.

BROOK, M. V. Ultrasonic Inspection Technology Development and Search Unit Design. Editora Wiley, ed.1, Nova York, 2012.

CHEEKE, J. D. N. Fundamentals and Applications of Ultrasonic Waves. Editora CRC Press, ed.1, Londres, 2002.

HIRAO, M. \& OGI, H. EMATs for Science and Industry. Editora Springer, 1a edição, Nova York , 2003.

LUZ, A. M. R. \& ÁlVARES, B. A. Física: volume 2. Editora Scipione S.A, São Paulo, 2005.

MARTIN, C. C. Ultrassom. Editora Associação Brasileira de Ensaios Não Destrutivos e Inspeção, 1a edição, São Paulo, 2012.

NISHIM OTO, K. Desafios do Pré-Sal para a Comunidade Científica e Tecnológica Brasileira. Revista USP, n. 95, p. 43 - 48, São Paulo, setembro / outubro / novembro 2012; 
NOGUEIRA, C. Ultrasonic Wave Propagation in Concrete. Editora Verlag Dr. Muller, 1a edição, Saarbrücken, 2009.

NUSSENZVEIG, H. M. Curso de física básica, volume 2. 4ae edição revisada, Editora Blücher, São Paulo, 2002.

OLIVEIRA, A. Pré-Sal: a Oportunidade e seus Desafios. Revista USP, n. 95, p. 23 32, São Paulo, setembro / outubro / novembro 2012;

OLYM PUS. Introduction to Phased Array Ultrasonic Technology Applications. $1^{\text {a }}$ edição, 2007. Disponível em: www.olympus.com.br, acessado em 20/02/2013, 376 páginas.

OLYM PUS. Phased Array Testing and Basic Theory for Industrial Applications. $1^{\mathrm{a}}$ edição, 2010. Disponível em: www.olympus.com.br, download em 20/02/2013, 122 páginas;

RICHTER, M. R. A Tecnologia do Ultrassom Computadorizado (Automático, Phased Array e ToFD e Aplicações Práticas na Indústria do Petróleo e Petroquímico no Brasil. In: Apresentação ao Comitê do Setor de Óleo e Gás da Associação Brasileira de Ensaios Não-Destrutivos e Inspeções, arquivo eletrônico cedido pelo autor, 115 p., 2013.

ROSE, J. L. Ultrasonic Waves in Solid Media. Editora Cambridge University Press, $1^{\text {a }}$ edição, Cambridge, 2004.

TELLES, P. C. S. Tubulações Industriais. Editora LTC. 10ae edição, São Paulo, 2010.

WEEKS, M. Processamento Digital de Sinais - Utilizando o MATLAB e Wavelets. Editora Gen - LTC, Tradução da 2a edição, São Paulo, 2012.

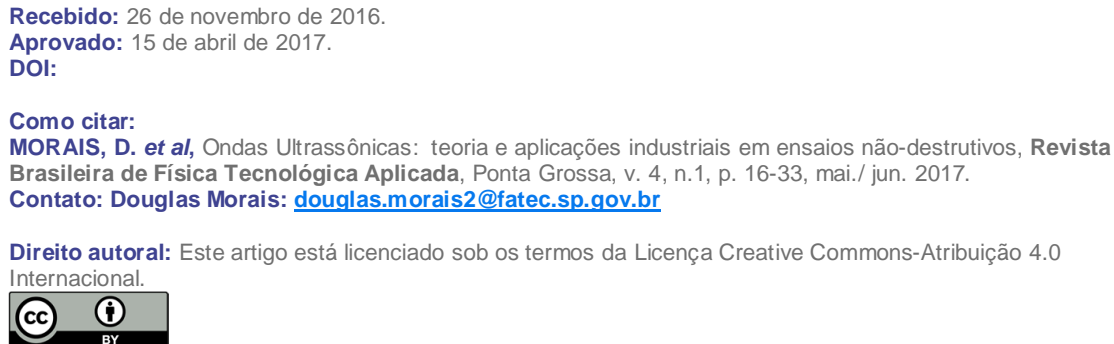

Direito autoral: Este artigo está licenciado sob os termos da Licença Creative Commons-Atribuição 4.0 Internacional.

(c) (i) 